\title{
Critics on "Adjunctive Memantine Therapy for Cognitive Impairment in Chronic Schizophrenia: A Placebo-Controlled Pilot Study"
}

\author{
Chi-Un Pae ${ }^{凶}$ \\ Department of Psychiatry, The Catholic University of Korea College of Medicine, Seoul, Republic of Korea \\ Department of Psychiatry and Behavioral Sciences, Duke University Medical Center, Durham, NC, USA
}

Dear Sir: I have read with great interest the paper by Lee and colleagues that investigated the effects of memantine, an $\mathrm{N}$-methyl-d-aspartate (NMDA) receptor antagonist, on cognitive impairments in Korean patients with chronic schizophrenia. ${ }^{1}$ According to the results, they failed to find any beneficial effects of memantine augmentation over placebo in cognitive functions and negative symptoms which were measured by various objective rating scales, such as Positive and Negative Symptom Scale (PANSS) and Korean Mini-Mental Status Examination (K-MMSE) in their double-blind, placebo-controlled, 12 weeks clinical trial. However, their study is the first one directly digging out the possible superiority of memantine augmentation over placebo in Asian patients with chronic schizophrenia, deserving add-up of more information for future studies.

The stem acceptable scientific backgrounds for their study may come from some encouraging evidence from previous animal and clinical trials, ${ }^{2-4}$ although disappointing result also exists like the authors' study result. ${ }^{5}$ The major shortcoming of their study should be the insufficient sample size to detect significant differences in cognitive functions and negative symptoms of schizophrenia between memantine augmenatation and placebo. The required sample size of their study should be 74 patients in total based on the results of K-MMSE difference of 2 points with standard deviation (SD) of 3 under $80 \%$ power

Received: August 12, 2012 Revised: October 23, 2012

Accepted: October 23, 2012 Available online: February 8, 2013

$\triangle$ Correspondence: Chi-Un Pae, MD

Department of Psychiatry, Bucheon St. Mary's Hospital, The Catholic University of Korea College of Medicine, 327 Sosa-ro, Wonmi-gu, Bucheon 420-717, Republic of Korea

Tel: +82-32-340-2140, Fax: +82-32-340-2255

E-mail: pae@catholic.ac.kr

(a) This is an Open Access article distributed under the terms of the Creative Commons Attribution Non-Commercial License (http://creativecommons.org/licenses/bync/3.0) which permits unrestricted non-commercial use, distribution, and reproduction in any medium, provided the original work is properly cited. (alpha $=0.05)$ between memantine augmentation and placebo; however, when we assume only $10 \%$ early dropout during such clinical trial, the final sample size should be almost 80 . Conversely, with their enrolled samples of 26, the detectable difference between memantine augmentation and placebo had to be 4 in K-MMSE score with SD of 3.5 under $80 \%$ power; hence, the sensitivity of primary and secondary outcomes of their study was not so much big. In this context, the findings from previous study ${ }^{5}$ also deliver clinicians a meaningful lesson. The sample size of Keefe's study ${ }^{6}$ was sufficient and 125 patients per treatment group were enrolled based on a twosided test with alpha of 0.05 and $80 \%$ power to show statistically significant differences between treatment arms [assuming between treatments effect size of 0.4 in the Clinical Antipsychotic Trials of Intervention Effectiveness (CATIE) neurocognitive battery composite score]; however, they also failed to prove donepezil's effect in patients with schizophrenia like both Dr Lee's ${ }^{1}$ and Lieberman's ${ }^{5}$ studies. Taken together, sensitivity and validity of classical neurocognitive batteries in schizophrenia should be re-evaluated and we may need more specific neuropsychological ratings relating treatment outcomes with drug therapy for patients with schizophrenia. Second, the subjects' characteristics are also slightly different compared to previous studies. Approximately 10 years are older in mean ages compared to previous studies. Conventional antipsychotics that may have more negative effects on cognitive functions were used in their study, while other studies that have shown positive effects of memantine on negative symptoms in patients with schizophrenia, adopted atypical antipsychotics. The antipsychotic dose in memantine group was numerically higher by $22 \%$ than placebo group; hence, the outcome results should be tested by ANCOVA to control the baseline effects. Their study was based on 8 multi-center, indicating the heterogeneity of samples that might also affect on negative study out- 
comes; they had to look at all data ratings across the multicenter whether some center had deviating effects on outcomes. In addition, the memantine augmentation may have no true efficacy on cognition and negative symptoms in Korean patients with schizophrenia since the changes in all primary and secondary outcome variables were completely negative in their study; strikingly, the changes in K-MMSE scores were less than $10 \%$, while it was $27 \%$ in the study by Dr. de Lucena and colleagues. ${ }^{2}$ Finally, a recent meta-analysis has proved that specific cognitive deficits (memory, and the motor speed and attention part of executive function) but not global cognitive functions were only responsive to adjuctive acetylcholinesterase inhibitors treatments in patients with schizophrenia and schizoaffective disorder. ${ }^{7}$ These findings may suggest differential and/or partial effects of cognitive enhancers such as memantine or donepezil in patients with schizophrenia unlike those with Alzheimer's disease.

In conclusion, availability of clear evidence from methodologically-more advanced and adequately-powered clinical trials will be mandatory to demonstrate whether or not memantine augmentation is efficacious in cognitive decline in patients with schizophrenia.

\section{REFERENCES}

1. Lee JG, Lee SW, Lee BJ, Park SW, Kim GM, Kim YH. Adjunctive memantine therapy for cognitive impairment in chronic schizophrenia: a placebo-controlled pilot study. Psychiatry Investig 2012;9:166-173.

2. de Lucena D, Fernandes BS, Berk M, Dodd S, Medeiros DW, Pedrini M, et al. Improvement of negative and positive symptoms in treatmentrefractory schizophrenia: a double-blind, randomized, placebo-controlled trial with memantine as add-on therapy to clozapine. J Clin Psychiatry 2009;70:1416-1423.

3. Krebs M, Leopold K, Hinzpeter A, Schaefer M. Neuroprotective agents in schizophrenia and affective disorders. Expert Opin Pharmacother 2006;7:837-848.

4. Krivoy A, Weizman A, Laor L, Hellinger N, Zemishlany Z, Fischel T. Addition of memantine to antipsychotic treatment in schizophrenia inpatients with residual symptoms: A preliminary study. Eur Neuropsychopharmacol 2008;18:117-121.

5. Lieberman JA, Papadakis K, Csernansky J, Litman R, Volavka J, Jia XD, et al. A randomized, placebo-controlled study of memantine as adjunctive treatment in patients with schizophrenia. Neuropsychopharmacology 2009;34:1322-1329.

6. Keefe RS, Malhotra AK, Meltzer HY, Kane JM, Buchanan RW, Murthy A, et al. Efficacy and safety of donepezil in patients with schizophrenia or schizoaffective disorder: significant placebo/practice effects in a 12week, randomized, double-blind, placebo-controlled trial. Neuropsychopharmacology 2008;33:1217-1228.

7. Ribeiz SR, Bassitt DP, Arrais JA, Avila R, Steffens DC, Bottino CM. Cholinesterase inhibitors as adjunctive therapy in patients with schizophrenia and schizoaffective disorder: a review and meta-analysis of the literature. CNS Drugs 2010;24:303-317. 